\title{
Seletividade e Absorção Radicular do Sulfentrazone em Clones de Eucalipto ${ }^{1}$
}

\author{
Sulfentrazone Selectivity and Root Absorption in Eucalyptus Clones
}

CARBONARI, C.A. ${ }^{2}$, VELINI, E.D. ${ }^{3}$, GOMES, G.L.G.C. ${ }^{4}$, TAKAHASHI, E.N. ${ }^{5}$ e ARALDI, R. ${ }^{4}$

\begin{abstract}
RESUMO - O objetivo deste trabalho foi avaliar a seletividade e a absorção do sulfentrazone em clones de eucalipto. O primeiro experimento foi instalado em casa de vegetação, em delineamento inteiramente casualizado, com quatro repetições, no esquema fatorial $2 \times 4$, sendo duas doses do sulfentrazone (400 e $600 \mathrm{~g} \mathrm{ha}^{-1}$ ) e quatro clones de eucalipto, híbridos de Eucalyptus grandis x E. urophylla (FB1, FB2, FB3 e FB4). Foram realizadas avaliações visuais de intoxicação das plantas de eucalipto e, no final do estudo, determinou-se a massa seca da parte aérea dos clones. No segundo experimento, foram utilizados os mesmos clones, sendo estes acondicionados em tubos falcon com $50 \mathrm{~mL}$ da solução contendo o sulfentrazone na concentração de $129 \mathrm{mM}$. As plantas de eucalipto permaneceram por 24 horas com as raízes imersas na solução e, em seguida, foi realizada a extração da seiva do xilema das plantas por meio de uma câmara de pressão. A concentração de sulfentrazone na seiva das plantas foi determinada através de cromatografia líquida e espectrometria de massas. O clone FB3 apresentou menor acúmulo de massa seca em relação aos demais, o que pode estar diretamente associado aos altos níveis de intoxicação observados. O clone FB2, apesar de mostrar elevada intoxicação, não apresentou níveis tão elevados de redução de massa seca em relação à testemunha. No tocante às concentrações de sulfentrazone nas plantas, elas foram proporcionais ao acúmulo de massa seca, o que indica que as variações na seletividade dos clones de eucalipto podem estar relacionadas à absorção diferenciada do herbicida.
\end{abstract}

Palavras-chave: Eucalyptus spp., fitointoxicação, herbicida.

\begin{abstract}
The objective of this work was to evaluate the selectivity and absorption of sulfentrazone in clones of eucalyptus. The first experiment was set up in a greenhouse in a completely randomized design in a factorial $2 \times 4$, with two rates of sulfentrazone (400 and $600 \mathrm{~g} \mathrm{ha}^{-1}$ ) and four clones of eucalyptus, hybrids of Eucalyptus grandis $x$ E. urophylla (FB1, FB2, FB3, FB4), with four replications. Visual evaluations of intoxication of the eucalyptus plants were carried out and the dry mass of the plants was determined at the end of the study. In the second experiment, the same clones of eucalyptus were placed in falcon tubes with $50 \mathrm{~mL}$ of sulfentrazone at a concentration of $129 \mathrm{Mm}$, remaining for 24 hours until the extraction of xylem sap plant through a pressure pump. The concentration of sulfentrazone in the sap of the plants was determined by liquid chromatography and mass spectrometry. Clone FB3 showed greater dry mass reduction in relation to the other clones, and this reduction can be directly associated with the high levels of phytotoxicity observed. Clone FB2 presented high levels of intoxication, but did not show high dry mass reduction. The concentrations of sulfentrazone absorbed by the plants were proportional to the reductions in dry mass, indicating that variations in the selective eucalyptus clones may be related to the differentiated absorption of the herbicide.
\end{abstract}

Keywords: Eucalyptus spp., phytointoxication, herbicide.

Recebido para publicação em 16.11.2010 e aprovado em 29.7.2011.

2 Professor Assistente, Dr., Dep. de Produção Vegetal, Faculdade de Ciências Agronômicas - FCA/UNESP, Caixa postal 237, 18603-970 Botucatu-SP; ${ }^{3}$ Professor Adjunto, Dep. de Produção Vegetal, FCA/UNESP; ${ }^{4}$ Enga-Agrạ., Aluna de Doutorado em Agronomia, FCA/UNESP; ${ }^{5}$ Engenheiro Florestal, M.Sc., Pesquisador Florestal, FIBRIA, Rod. MS395, Caixa Postal 515, Três Lagoas-MS.

Planta Daninha, Viçosa-MG, v. 30, n. 1, p. 147-153, 2012 


\section{INTRODUÇÃO}

O florestamento com eucalipto no Brasil está em plena expansão, com aproximadamente 4,5 milhões de hectares plantados em 2010 e crescimento médio de $7,1 \%$ nos últimos cinco anos. Essa expansão é resultado de um conjunto de fatores, como o rápido crescimento, a alta produtividade e o direcionamento de novos investimentos por parte de empresas desses segmentos que utilizam a madeira como matéria-prima em processos industriais (ABRAF, 2010).

O manejo de plantas daninhas na cultura do eucalipto assume destaque entre os tratos culturais, apresentando reflexos diretos no rendimento e nos custos de produção, sendo uma das atividades mais onerosas e um componente muito importante desde a fase inicial do ciclo até o estabelecimento do povoamento florestal, onde estas passam a dominar as demais espécies vegetais do local (Toledo et al., 2003).

O manejo das plantas daninhas em florestamentos, nas diversas etapas do seu processo produtivo, é realizado, basicamente, pelo emprego de métodos mecânicos e químicos, isolados ou combinados (Toledo et al., 2003). No método químico, entre os herbicidas utilizados, destaca-se o sulfentrazone, absorvido pelas plantas através da raiz e das folhas, com movimento limitado no floema em função da rápida dessecação foliar (Rodrigues \& Almeida, 2005). Esse produto inibe a protoporfirinogênio oxidase (PROTOX), causando o acúmulo de protoporfirinogênio IX e formando o oxigênio singleto, que é responsável pela peroxidação das membranas (Scalla et al., 1990). Além do acúmulo de protoporfirina IX e produção de oxigênio singleto, a produção de clorofila é inibida pelo bloqueio da rota (Duke et al., 1991).

O principal fator para a tolerância de algumas plantas ao sulfentrazone parece ser o metabolismo diferencial, embora outros efeitos, como o retardo do desenvolvimento de pelos radiculares em Cassia ocidentalis e o dano celular diferencial em cultivares de soja, também sejam fatores importantes (Dayan et al., 1996, 1997), além do movimento limitado desse herbicida a partir do local de aplicação até o sítio de ação, das modificações moleculares desse sítio e da rápida metabolização (Vaughn \& Duke, 1991). De acordo com Bailey et al. (2003), a absorção radicular e a translocação diferencial contribuem para niveis diferenciados de tolerância, como os mecanismos primários, em batata, Chenopodium album e Datura stramonium.

Cultivares de soja apresentam diferentes níveis de tolerância ao sulfentrazone aplicado em pré-emergência da cultura (Dayan et al., 1997; Swantek et al., 1998). Dayan et al. (1997) não encontraram diferenças na absorção e translocação de ${ }^{14} \mathrm{C}$-sulfentrazone, e pequenas diferenças no metabolismo foram observadas até três horas após os tratamentos, comparando cultivares sensiveis e tolerantes. Esses autores relataram que a tolerância está associada à capacidade dos cultivares tolerantes ao herbicida em metabolizar o estresse peroxidativo, potencialmente através de sistemas antioxidantes (Finckh \& Kunert, 1985). Velini et al. (2005) verificaram que configurações da enzima Protox ou promotores que permitiriam diferentes níveis de expressão podem permitir genótipos que sejam mais tolerantes aos herbicidas que atuam na inibição dessa enzima.

Para o eucalipto, são observados clones com diferentes niveis de tolerância ao sulfentrazone, e possivelmente isso esteja relacionado com as quantidades absorvidas desse herbicida. O objetivo deste trabalho foi avaliar a seletividade e absorção radicular do sulfentrazone em clones de eucalipto.

\section{MATERIAL E MÉTODOS}

Foram realizados dois experimentos no Núcleo de Pesquisas Avançadas em Matologia (NUPAM), na Faculdade de Ciências Agronômicas, UNESP, campus de Botucatu, onde foi avaliada a tolerância de quatro clones de eucalipto ao herbicida sulfentrazone, além da absorção radicular do herbicida por eles.

O primeiro experimento foi instalado em delineamento inteiramente casualizado, com quatro repetições, no esquema fatorial $2 \times 4$, sendo duas doses de sulfentrazone (400 e $600 \mathrm{~g} \mathrm{ha}^{-1}$ ) e quatro clones híbridos de Eucalyptus grandis x E. urophylla (FB1, FB2, FB3 e FB4), pertencentes à empresa Fibria Celulose. Foram mantidas plantas dos 
diferentes clones sem aplicação do herbicida, as quais serviram detestemunhas na avaliação dos sintomas causados pelo herbicida nas plantas tratadas.

O experimento foi conduzido em casa de vegetação, em vasos plásticos preenchidos com 10 L de solo, com teor de matéria orgânica de $14 \mathrm{~g} \mathrm{dm}^{-3}$, pH de 6,4 e textura arenosa (84\% de areia, $11 \%$ de argila e 5\% de silte).

A aplicação do herbicida foi feita com pulverizador estacionário em laboratório, equipado com quatro pontas de pulverização da série DG 11002, espaçadas de $50 \mathrm{~cm}$, com pressão de $200 \mathrm{KPa}$ e volume de calda de $200 \mathrm{~L} \mathrm{ha}^{-1}$. O sulfentrazone foi pulverizado diretamente sobre as unidades experimentais; em seguida, foram plantadas as mudas dos clones de eucalipto.

Foram realizadas avaliações visuais aos 7, 21, 35, 49, 63 e 77 dias após a aplicação dos tratamentos, adotando-se uma escala percentual de notas, em que 0 significa ausência total de intoxicação e 100 a morte das plantas.

Aos 90 dias após a aplicação do sulfentrazone, foi seccionada a parte aérea das plantas ao nivel do solo, acondicionadas em sacos de papel e, posteriormente, levadas para secagem em estufa de circulação forçada de ar a $60{ }^{\circ} \mathrm{C}$. Após a secagem da parte aérea das plantas, foi realizada a pesagem da massa seca de cada uma delas, em balança de 0,0001 g de precisão.

O segundo experimento foi instalado com o objetivo de quantificar a absorção radicular do sulfentrazone. Assim, visando comparar a concentração do sulfentrazone na seiva do xilema dos quatro clones avaliados, foram utilizadas seis plantas de cada clone de eucalipto (repetições). As mudas de eucalipto foram retiradas dos tubetes com substrato e, a seguir, tiveram suas raízes lavadas. Após esse procedimento, as raizes das mudas de eucalipto foram acondicionadas em tubos falcon com $50 \mathrm{~mL}$ da solução contendo o sulfentrazone na concentração de 129 mM, nos quais foram mantidas por 24 horas, permitindo a absorção radicular do herbicida pelas plantas. Foi utilizada uma única concentração do herbicida sulfentrazone, que permitiu um comparativo entre os niveis do herbicida absorvido pelos diferentes clones. Essa concOentração foi selecionada em função da quantificação do sulfentrazone na solução do solo em áreas cultivadas com eucalipto (Velini et al., 2010) e dos teores de sulfentrazone no solo encontrados por Ohmes et al. (2000) e Reddy et al. (1998).

Após esse período, as plantas foram cortadas ao nivel do solo, e a parte aérea foi acondicionada em uma bomba de Scholander, para extração da seiva do xilema (Scholander et al., 1965). A pressão incidida na câmara foi aplicada até culminar em gotas de seiva na superfície do corte (Liang et al., 1996). Com o auxílio de uma pipeta Pasteur, a seiva foi coletada no caule das plantas e acondicionada em vial, para posterior determinação dos níveis de sulfentrazone absorvido pelas plantas no período. Para as determinações dos niveis de sulfentrazone nas plantas e na solução dos tubos falcon, foi desenvolvido um método analítico em cromatógrafo líquido da marca Shimadzu, equipado com software Class VP 6.0, bomba quaternária LC 20 AD, degaseificador DGU 20AS, injetor automático SIL $10 \mathrm{AF}$, forno CTO 10ASVP e detector de massas LCMS-2010 EV. Para as análises cromatográficas, foi utilizada uma coluna de C18, marca Synergi $2.5 \mu$ Hydro-RP, dimensões de 50 x 4,6 mm, com volume de injeção de $30 \mu \mathrm{L}$. O tempo total de corrida foi de 12,5 minutos, e o tempo de retenção do sulfentrazone, de 2,8 minutos. Foram estabelecidos seis pontos para a curva de calibração, sendo empregada a quantificação em diferentes concentrações dos padrões de sulfentrazone. No desenvolvimento do método foi utilizado o padrão analítico com grau de pureza certificada de 99,8\%.

Os resultados da intoxicação visual das plantas de eucalipto foram submetidos à análise de variância pelo teste $\mathrm{F}$, e as médias, comparadas pelo teste de Tukey, sendo os dois testes efetuados a $5 \%$ de probabilidade. Os resultados de massa seca e da concentração de sulfentrazone na seiva das plantas de eucalipto foram analisados estabelecendo-se o intervalo de confiança pelo teste $\mathrm{T}$ a $10 \%$ de probabilidade. Para determinar o intervalo de confiança, foi utilizada a seguinte equação: IC $=(\mathrm{t} x$ desvpad $) /$ raiz $\mathrm{nr}$, em que $\mathrm{IC}=$ intervalo de confiança; $\mathrm{T}=$ valor de $\mathrm{T}$ tabelado, em nivel de $10 \%$ de probabilidade; desvpad = desviopadrão; e raiz nr = raiz quadrada do número de repetições. 


\section{RESULTADOS E DISCUSSÃO}

Na Tabela 1 são apresentados os resultados de intoxicação dos clones de eucalipto aos 7, 21 e 35 dias após a aplicação (DAA) do sulfentrazone. Aos 7 DAA foram observados os primeiros sintomas de intoxicação das plantas de eucalipto; o clone FB1 apresentou os menores niveis de intoxicação para as duas doses testadas, e o clone FB3, os maiores niveis de intoxicação para a menor dose de sulfentrazone. Já os clones FB2 e FB4 mostraram resultados semelhantes para ambas as doses do herbicida. Foram observados como principais sintomas: folhas arroxeadas com necroses, deformações destas e perda da dominância apical em algumas plantas. Esses sintomas são semelhantes aos descritos por Takahashi et al. (2009) ao relatarem que os sintomas do sulfentrazone iniciaram-se a partir do sétimo dia após a aplicação. Velini et al. (2005) afirmam que o sulfentrazone pode ser tóxico para o eucalipto e que as folhas jovens formadas entre o plantio e a aplicação demonstram maior sensibilidade ao produto, podendo apresentar lesões bastante acentuadas.

Aos 21 DAA, os clones demonstraram sintomas visuais de intoxicação bastante semelhantes, exceto o FB3 para a maior dose de sulfentrazone, onde as injúrias foram mais severas (Tabela 1). Aos 35 DAA também se observaram niveis de intoxicação semelhantes para os diferentes clones na dose de $400 \mathrm{~g} \mathrm{ha}^{-1}$. No entanto, quando foram aplicados $600 \mathrm{~g} \mathrm{ha}^{-1}$, os clones FB2 e FB3 apresentaram níveis mais elevados de intoxicação.

Aos 49 DAA, os clones testados não apresentaram diferenças nos niveis de intoxicação na menor dose testada. No entanto, os clones FB2 e FB3 mostraram sintomas mais elevados de intoxicação, respectivamente de 30 e $43 \%$, para a maior dose de sulfentrazone (Tabela 2). Aos 63 DAA, o clone FB4 apresentou os menores niveis de intoxicação, independentemente da dose utilizada. Os clones que demonstraram maior sensibilidade ao sulfentrazone novamente foram o FB2 e FB3. Também aos 77 DAA foram observados resultados semelhantes quanto às respostas dos clones, porém nessa avaliação observam-se reduções nos níveis de intoxicação em relação ao período avaliado anteriormente. Segundo Rodrigues \& Almeida, (2005) o sulfentrazone apresenta elevada persistência no solo, sendo sua meia-vida em solos brasileiros de 180 dias. Ainda segundo esses autores, o produto apresenta solubilidade em água de $490 \mathrm{mg} \mathrm{L}{ }^{1}$ e mobilidade moderada, com baixa adsorção. Essas características do sulfentrazone ajudam a explicar a persistência dos efeitos nos diferentes clones de eucalipto, mesmo aos 77 DAA.

Os resultados demonstraram que todos os clones de eucalipto testados paralisaram o

Tabela 1 - Intoxicação (\%) de clones de eucalipto aos 7, 21 e 35 dias após a aplicação (DAA) do sulfentrazone. Botucatu-SP, 2010

\begin{tabular}{|c|c|c|c|c|}
\hline \multirow{3}{*}{ Dose $\left(\mathrm{g} \mathrm{ha}^{-1}\right)$} & \multicolumn{4}{|c|}{ Clone de eucalipto } \\
\hline & FB1 & FB2 & FB3 & FB4 \\
\hline & \multicolumn{4}{|c|}{$7 \mathrm{DAA}$} \\
\hline 400 & $4,3 \mathrm{Aa}$ & $6,8 \mathrm{Aab}$ & $13,8 \mathrm{Ab}$ & $9,8 \mathrm{Aab}$ \\
\hline 600 & $6,3 \mathrm{Aa}$ & $14,5 \mathrm{Bb}$ & $14,8 \mathrm{Ab}$ & $14,25 \mathrm{Ab}$ \\
\hline $\mathrm{F}$ (clone) & \multicolumn{4}{|c|}{$8,014^{* *}$} \\
\hline $\mathrm{F}(\mathrm{dos})$ & \multicolumn{4}{|c|}{$8,065^{* *}$} \\
\hline $\mathrm{F}$ (clone $\mathrm{x}$ dose) & \multicolumn{4}{|c|}{$1,248^{\text {ns }}$} \\
\hline $\mathrm{CV}(\%)$ & \multicolumn{4}{|c|}{36,17} \\
\hline DMS (clone) & \multicolumn{4}{|c|}{5,56} \\
\hline DMS (dose) & \multicolumn{4}{|c|}{7,43} \\
\hline & \multicolumn{4}{|c|}{$21 \mathrm{DAA}$} \\
\hline 400 & $10,3 \mathrm{Aa}$ & $13,8 \mathrm{Aa}$ & $18,00 \mathrm{Aa}$ & $14,5 \mathrm{Aa}$ \\
\hline 600 & $15,0 \mathrm{Aa}$ & $17,5 \mathrm{Aa}$ & $32,00 \mathrm{Bb}$ & $19,5 \mathrm{Aa}$ \\
\hline F (clone) & \multicolumn{4}{|c|}{$19,62 * *$} \\
\hline $\mathrm{F}($ dose $)$ & \multicolumn{4}{|c|}{$111,59^{* *}$} \\
\hline $\mathrm{F}$ (clone $\mathrm{x}$ dose $)$ & \multicolumn{4}{|c|}{$2,37^{\text {ns }}$} \\
\hline $\mathrm{CV}(\%)$ & \multicolumn{4}{|c|}{26,00} \\
\hline DMS (clone) & \multicolumn{4}{|c|}{6,41} \\
\hline DMS (dose) & \multicolumn{4}{|c|}{8,56} \\
\hline & \multicolumn{4}{|c|}{$35 \mathrm{DAA}$} \\
\hline 400 & $13,5 \mathrm{Aa}$ & $11,8 \mathrm{Aa}$ & $15,0 \mathrm{Aa}$ & $11,3 \mathrm{Aa}$ \\
\hline 600 & $15,0 \mathrm{Aa}$ & $19,5 \mathrm{Ba}$ & $35,0 \mathrm{Bb}$ & $14,5 \mathrm{Aa}$ \\
\hline F (clone) & \multicolumn{4}{|c|}{$54,89^{* *}$} \\
\hline $\mathrm{F}(\mathrm{dose})$ & \multicolumn{4}{|c|}{$25,11 * *$} \\
\hline $\mathrm{F}$ (clone $\mathrm{x}$ dose $)$ & \multicolumn{4}{|c|}{$14,49^{* *}$} \\
\hline $\mathrm{CV}(\%)$ & \multicolumn{4}{|c|}{18,30} \\
\hline DMS (clone) & \multicolumn{4}{|c|}{4,52} \\
\hline DMS (dose) & \multicolumn{4}{|c|}{6,04} \\
\hline
\end{tabular}

Médias seguidas de mesma letra, maiúscula na coluna e minúscula na linha, não diferem entre si pelo teste de Tukey $(\mathrm{p}<0,05)$.

$*$, ** significativo a 5 e $1 \%$ de probabilidade, respectivamente, e ${ }^{\text {ns }}$ não significativo. 
acúmulo de massa seca da parte aérea aos 77 DAA, quando submetidos à aplicação do sulfentrazone, sendo esse dano maior para a dose de $600 \mathrm{~g} \mathrm{ha}^{-1}$ (Figura 1). Pode-se observar que o clone FB3 apresentou os menores acúmulos de massa seca para as doses de sulfentrazone testadas, com $38 \%$ de perda de massa seca para a maior dose. Os clones FB1 e FB2 apresentaram niveis intermediários de perdas de massa seca da parte aérea na maior dose, porém na menor dose o primeiro foi menos sensivel. O clone FB4 apresentou maior acúmulo de massa seca da parte aérea, demonstrando menor sensibilidade ao herbicida sulfentrazone.

Tabela 2 - Intoxicação (\%) de clones de eucalipto aos 49, 63 e 77 dias após a aplicação (DAA) de sulfentrazone. Botucatu-SP, 2010

\begin{tabular}{|c|c|c|c|c|}
\hline \multirow{3}{*}{ Dose $\left(\mathrm{g} \mathrm{ha}^{-1}\right)$} & \multicolumn{4}{|c|}{ Clone de eucalipto } \\
\hline & FB1 & FB2 & FB3 & FB4 \\
\hline & \multicolumn{4}{|c|}{$49 \mathrm{DAA}$} \\
\hline 400 & $18,5 \mathrm{Aa}$ & $14,0 \mathrm{Aa}$ & $20,8 \mathrm{Aa}$ & $15,8 \mathrm{Aa}$ \\
\hline 600 & $24,8 \mathrm{Aab}$ & $30,0 \mathrm{Bb}$ & $43,0 \mathrm{Bc}$ & $16,5 \mathrm{Aa}$ \\
\hline F (clone) & \multicolumn{4}{|c|}{$55,56 * *$} \\
\hline $\mathrm{F}($ dose $)$ & \multicolumn{4}{|c|}{$18,65^{* *}$} \\
\hline $\mathrm{F}$ (clone $\mathrm{x}$ dose) & \multicolumn{4}{|c|}{$10,9^{* *}$} \\
\hline $\mathrm{CV}(\%)$ & \multicolumn{4}{|c|}{18,74} \\
\hline DMS (clone) & \multicolumn{4}{|c|}{6,26} \\
\hline DMS (dose) & \multicolumn{4}{|c|}{8,38} \\
\hline & \multicolumn{4}{|c|}{$63 \mathrm{DAA}$} \\
\hline 400 & $22,0 \mathrm{Ab}$ & $29,5 \mathrm{Ac}$ & 19,0 Aab & $14,5 \mathrm{Aa}$ \\
\hline 600 & $28,5 \mathrm{Bb}$ & $39,5 \mathrm{Bc}$ & $39,8 \mathrm{Bc}$ & $17,3 \mathrm{Aa}$ \\
\hline F (clone) & \multicolumn{4}{|c|}{$60,09^{* *}$} \\
\hline $\mathrm{F}($ dose $)$ & \multicolumn{4}{|c|}{$37,35^{* *}$} \\
\hline $\mathrm{F}$ (clone $\mathrm{x}$ dose) & \multicolumn{4}{|c|}{$9,03 * *$} \\
\hline $\mathrm{CV}(\%)$ & \multicolumn{4}{|c|}{13,90} \\
\hline DMS (clone) & \multicolumn{4}{|c|}{5,32} \\
\hline DMS (dose) & \multicolumn{4}{|c|}{7,11} \\
\hline & \multicolumn{4}{|c|}{$77 \mathrm{DAA}$} \\
\hline 400 & $19,5 \mathrm{Ab}$ & $24,3 \mathrm{Ac}$ & $10,0 \mathrm{Aa}$ & $10,5 \mathrm{Aa}$ \\
\hline 600 & $20,0 \mathrm{Ab}$ & $28,8 \mathrm{Bc}$ & $28,0 \mathrm{Bc}$ & $13,5 \mathrm{Aa}$ \\
\hline $\mathrm{F}$ (clone) & \multicolumn{4}{|c|}{$64,13^{* *}$} \\
\hline $\mathrm{F}($ dose $)$ & \multicolumn{4}{|c|}{$53,33^{* *}$} \\
\hline $\mathrm{F}$ (clone $\mathrm{x}$ dose) & \multicolumn{4}{|c|}{$23,33^{* *}$} \\
\hline $\mathrm{CV}(\%)$ & \multicolumn{4}{|c|}{11,89} \\
\hline DMS (clone) & \multicolumn{4}{|c|}{3,35} \\
\hline DMS (dose) & \multicolumn{4}{|c|}{4,48} \\
\hline
\end{tabular}

Médias seguidas de mesma letra, maiúscula na coluna e minúscula na linha, não diferem entre si pelo teste de Tukey $(p<0,05)$.

*,** significativo a 5 e $1 \%$ de probabilidade, respectivamente, e ${ }^{n s}$ não significativo.
Quanto às respostas diferenciadas dos clones de eucalipto avaliados, fato semelhante foi obtido por Silva et al. (1994) ao verificarem respostas alternadas de espécies de eucalipto ao herbicida oxyfluorfen, o qual também apresenta como mecanismo de ação a inibição da Protox. Esses autores relataram ainda que $E$. grandis e E. saligna foram mais tolerantes à aplicação do oxyfluorfen, em relação a E. camaldulensis (intermediária) e E. citriodora (mais sensivel), demonstrando assim que a tolerância ao herbicida ocorre para as diferentes espécies do gênero Eucalyptus.

Takahashi et al. (2009), ao avaliarem os efeitos da aplicação de doses de sulfentrazone em dois clones de eucalipto, observaram que os materiais genéticos mostraram comportamentos distintos para área foliar total e massa seca de folhas. Esses parâmetros são importantes para os processos bioquímicos e fisiológicos da planta e podem implicar diferentes niveis de desenvolvimento, ou mesmo de qualidade da madeira. Para o pinhão-manso, Rocha et al. (2010) observaram que genótipos da cultura apresentaram niveis variáveis de tolerância ao herbicida sulfentrazone.

Na Figura 2 estão apresentados os resultados da concentração do sulfentrazone na seiva extraída do xilema dos clones de eucalipto. Observa-se que o clone FB3 apresentou concentração bastante superior à dos demais clones, o que indica que ele tem capacidade de absorver maior quantidade de sulfentrazone. Esse resultado corrobora os apresentados na Figura 1, uma vez que esse foi o clone que apresentou menores taxas de acúmulo de matéria seca das plantas. Observa-se ainda que o clone FB4 apresentou também nível de concentração superior ao dos demais (acima de $3 \mathrm{mg} \mathrm{L}^{-1}$ ), o que também está de acordo com a Figura 1, onde este clone apresentou o segundo menor acúmulo de massa seca. Os demais clones mostraram niveis similares ou menores do sulfentrazone na seiva das plantas, o que é mais um indicativo da maior tolerância aos efeitos da aplicação do herbicida. $\mathrm{O}$ fato de os genótipos expressarem diferenças na sensibilidade a herbicidas pode estar ligado a fatores fisiológicos (Takahashi et al., 2009), como a translocação de fotoassimilados e o potencial de pressão no xilema, podendo influenciar a absorção e translocação de herbicidas 


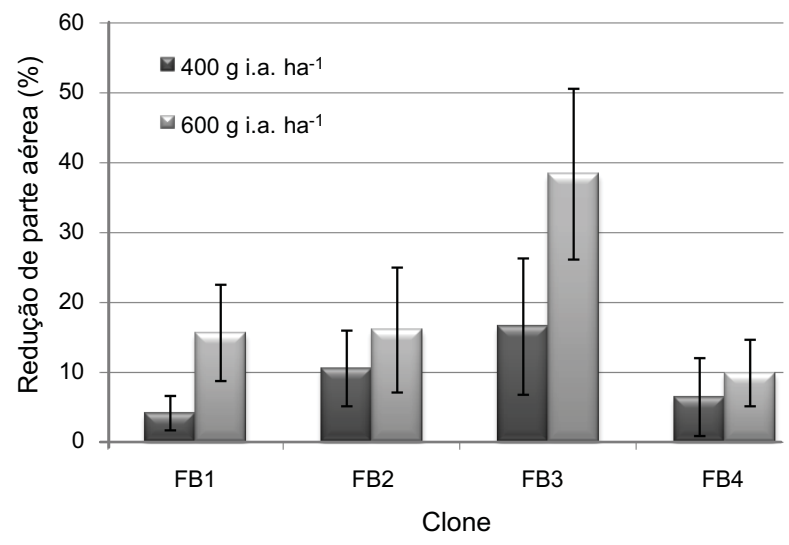

Figura 1 - Redução da massa seca da parte aérea (\%) de clones de eucalipto submetidos à aplicação de doses de sulfentrazone. Botucatu-SP, 2010.

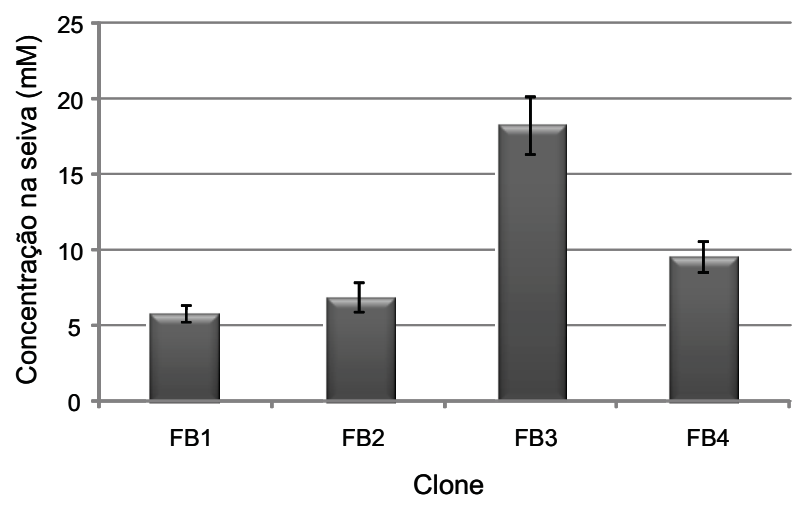

Figura 2 - Concentração de sulfentrazone (mM) na seiva do xilema das plantas de eucalipto submetidas à aplicação do herbicida. Botucatu-SP, 2010.

(King \& Radosevich, 1985). Isso pode originar variações na resposta de cada espécie para as aplicações de herbicidas (Paley \& Radosevich, 1984). Li et al. (2000) observaram que o cultivar de soja tolerante absorveu menos ${ }^{14} \mathrm{C}$ sulfentrazone antes da emergência do que o cultivar sensivel e concluíram que a tolerância ao sulfentrazone deveu-se principalmente à absorção diferencial. Machado et al. (2009), ao avaliarem a absorção foliar do glyphosate em dois clones de eucalipto, observaram níveis de absorção do produto bastante distintos, o que reflete a tolerância diferencial ao herbicida pelos diferentes clones.

O experimento foi instalado com uma condição de dose única, a qual foi disponibilizada diretamente em uma solução para absorção radicular. No entanto, no solo, a sorção e consequentemente a disponibilidade do sulfentrazone são bastante alteradas por diversos fatores relacionados ao solo, como textura, teor de matéria orgânica e $\mathrm{pH}$, e pelas condições ambientais, principalmente quanto à disponibilidade hídrica. Grey et al. (1997) avaliaram a sorção do herbicida sulfentrazone em diferentes solos e $\mathrm{pH}$ e observaram que ela geralmente diminui em resposta ao aumento do $\mathrm{pH}$, sobretudo quando os valores excedem o pKa do herbicida. Monqueiro et al. (2010) também observaram menor sorção do sulfentrazone em solo com pH mais elevado e também maior disponibilidade desse herbicida para solo de textura média, em relação a um solo argiloso. Resultado semelhante foi observado por Melo et al. (2010). Segundo estes autores, as características de solos florestais, nos quais grande quantidade de resíduos e matéria orgânica é encontrada, podem influenciar de forma bastante significativa a disponibilidade e a lixiviação de herbicidas aplicados no solo. Dessa forma, a quantidade de produto disponivel para a absorção e, consequentemente, para a intoxicação das plantas é bastante variável em campo.

Pode-se observar que o clone FB3 apresentou maior redução de massa seca em relação aos demais, e isso pode estar diretamente associado aos altos niveis de intoxicação. O clone FB2, apesar de demonstrar elevada intoxicação, não apresentou niveis consideráveis de redução do acúmulo de massa seca. Com relação aos niveis de sulfentrazone absorvidos pelas plantas, eles foram proporcionais ao acúmulo de massa seca observado, o que indica que as variações na seletividade dos clones de eucalipto podem estar relacionadas à absorção diferenciada do herbicida.

\section{LITERATURA CITADA}

\section{ASSOCIAÇÃOBRASILEIRA DE PRODUTORES DE FLORESTAS PLANTADAS - ABRAF.}

Anuário estatístico: ano base 2009. Brasília: 2010. $140 \mathrm{p}$.

BAILEY, W. A. et al. Absorption, translocation, and metabolism of sulfentrazone in potato and selected weed species. Weed Sci., v. 51, n. 1, p. 32-36, 2003. 
DAYAN, F. E.; WEETE, J. D.; HANCOCK, H. G. Physiological basis for differential sensitivity to sulfentrazone by sicklepod (Senna obtusifolia) and coffee senna (Cassia occidentalis). Weed Sci., v. 44, n. 1, p. 12-17, 1996.

DAYAN, F. E. et al. Soybean (Glycine max) cultivar differences in response to sulfentrazone. Weed Sci., v. 45, n. 5, p. 634-641, 1997.

DUKE, S. O. et al. Protoporphyrinogen oxidase inhibiting herbicides. Weed Sci., v. 39, n. 3, p. 465-473, 1991.

FINCKH, B. F.; KUNERT, K. J. Vitamin-C and vitamin-E: an antioxidative system against induced lipid peroxidation in higher plants. J. Agric. Food Chem., v. 33, n. 4, p. 574-577, 1985.

GREY, T. L.; WALKER, R. H.; HANCOCK, H. G. Sulfentrazone adsorption and mobility as affected by soil and pH. Weed Sci., v. 45, n. 5, p. 733-738, 1997.

KING, S. P.; RADOSEVICH, S. R. Herbicide tolerance in relation to growth and stress in conifers. Weed Sci., v. 33, n. 4, p. 472-478, 1985.

LIANG, J.; ZHANG, J.; WONG, M. H. Stomatal conductance in relation to xylem sap ABA concentration in two tropical trees: Acacia confuse and Litsea glutinosa. Plant Cell Environ., v. 19, n. 1, p. 93-100, 1996.

LI, Z.; WEHTJE, G. R.; WALKER, R. H. Physiological basis for the differential tolerance of Glycine max to sulfentrazone during seed germination. Weed Sci., v. 48, n. 2, p. 281-285, 2000.

MACHADO, A. F. L. et al. Absorção, translocação e exsudação radicular de glyphosate em clones de eucalipto: clones. Planta Daninha, v. 27, n. 3, p. 549-554, 2009.

MELO, C. A. D. et al. Lixiviação de sulfentrazone, isoxaflutole e oxyfluorfen no perfil de três solos.

Planta Daninha, v. 28, n. 2, p. 385-392, 2010.

MONQUERO, P. A. et al. Lixiviação e persistência dos herbicidas sulfentrazone e imazapic. Planta Daninha, v. 28, n. 1, p. 185-195, 2010.

OHMES, G. A.; HAYES, R. M.; MUELLER, T. C. Sulfentrazone dissipation in a Tennessee soil. Weed Technol., v. 14, n. 1, p. 100-105, 2000.
PALEY, S. M.; RADOSEVICH, S. R. Effect of physiological status and growth of ponderosa pine (Pinus ponderosa) and greenleaf manzanita (Arctostaphylos patula) on herbicide selectivity. Weed Sci., v. 32, n. 3, p. 395-402, 1984.

REDDY, K. N.; LOCKE, M.A. Sulfentrazone sorption, desorption, and mineralization in soils from two tillage systems. Weed Sci., v. 46, n. 4, p. 494-500, 1998.

ROCHA, P. R. R et al. Seletividade de herbicidas préemergentes ao pinhão-manso (Jatropha curcas).

Planta Daninha, v. 28, n. 4, p. 801-806, 2010.

RODRIGUES, B. N.; ALMEIDA, F. S. Guia de herbicidas. 5.ed. Londrina: Edição dos Autores, 2005.

SCALLA, R. et al. Recent advances in the mode of action of diphenyl ether and related herbicides.

Zeitschrift Naturforschung, v. 45, n. 5, p. 503-511, 1990.

SCHOLANDER, P. F. et al. Sap pressure in vascular plants. Science, v. 148, n. 3, p. 339-346, 1965.

SILVA, W. et al. Tolerância de Eucalyptus spp. a diferentes herbicidas. R. Árvore, v. 18, n. 3, p. 287-300, 1994.

SWANTEK, J. M.; SNELLER, C. H.; OLIVER, L. R. Evaluation of soybean injury from sulfentrazone and inheritance of tolerance. Weed Sci., v. 46, n. 3, p. 271-277, 1998.

TAKAHASHI, E. N. et al. Consequências da deriva de clomazone e sulfentrazone em clones de E. grandis X E. urophylla. R. Árvore, v. 33, n. 4, p. 685-683, 2009.

TOLEDO, R. E. B. et al. Faixas de controle de plantas daninhas e seus reflexos no crescimento de plantas de eucalipto. Sci. For., n. 64, p. 78-92, 2003.

\section{VAUGHN, K. C.; DUKE, S. O. Mechanisms of}

resistance. In: EBING, W. Chemistry of plant protection. New York: Springer-Verlag, 1991. p. 142-169.

VELINI, E. D. et al. Eucalyptus ESTs corresponding to the protoporphyrinogen IX oxidase enzyme related to the synthesis of heme, chlorophyll, and to the action of herbicides. Genetics Molec. Biol., v. 28, n. 3, p.555-561, 2005.

VELINI, E. D. et al. Disponibilidade no solo e eficácia do sulfentrazone no controle de plantas daninhas em área de produção de eucalipto. In: CONGRESSO BRASILEIRO DA CIÊNCIADAS PLANTAS DANINHAS, 27., Ribeirão Preto, 2010. Anais... Ribeirão Preto: 2010. p. 2734-2737.

Planta Daninha, Viçosa-MG, v. 30, n. 1, p. 147-153, 2012 